\title{
Defining the expressed breast cancer kinome
}

\author{
Alicia A Midland ${ }^{1}$, Martin C Whittle², James S Duncan², Amy N Abell², Kazuhiro Nakamura², Jon S Zawistowski², \\ Lisa A Carey ${ }^{3}$, H Shelton Earp III ${ }^{3}$, Lee M Graves², Shawn M Gomez ${ }^{1}$, Gary L Johnson² \\ ${ }^{1}$ Biomedical Engineering and Curriculum in Bioinformatics and Computational Biology; ${ }^{2}$ Department of Pharmacology; ${ }^{3}$ Depart- \\ ment of Medicine; Lineberger Comprehensive Cancer Center, University of North Carolina School of Medicine, Chapel Hill, NC \\ 27599, USA \\ Cell Research (2012) 22:620-623. doi:10.1038/cr.2012.25; published online 7 February 2012
}

Protein kinases are arguably the most tractable candidates for development of new therapies to treat cancer. Deep sequencing of breast cancer cell lines indicates each express 375 or so kinases, representing nearly $75 \%$ of the kinome. A rich network both downstream and upstream from key oncogenic kinases includes both tyrosine and serine/threonine kinases, giving plasticity and resiliency to the cancer cell kinome.

Protein kinases have proven to be highly tractable candidates for development of new cancer therapies with over 130 kinase-specific inhibitors currently in Phase 1-3 clinical trials [1]. Approximately 518 protein kinases are encoded by the human genome, collectively referred to as the kinome. Many kinases regulate normal growth and development while an intensely-studied small subset of kinases is dysregulated by multiple mechanisms and contribute to human cancer progression [2]. Several of these kinases (e.g. BCR-ABL, EGFR, ERBB2 (HER2), KIT, VEGFR, ALK, $\mathrm{BRAF}$, etc.) are proven therapeutic targets to treat cancer.

A rich network downstream and upstream from these key oncogenic

\footnotetext{
Correspondence: Gary L Johnson

Tel: +1-919-843-3107; Fax: +1-919-966-5640

E-mail: glj@med.unc.edu
}

kinases include both serine/threonine and tyrosine kinases, many of which are understudied and untargeted by chemical probes [3]. A key question in the development of new cancer therapeutics is which, if any, of these understudied kinases are potential therapeutic targets? Experimentally, the development of new effective kinase inhibitors is hindered by the fact that kinase networks are interrelated; inhibition by one pharmacologic kinase inhibitor has consequences beyond its cognate targets. The kinome is resilient and is often able to bypass inhibition of specific kinases by activation of other kinases, effectively resulting in resistance to single agents. Thus, rationally devising novel kinase inhibitor therapies requires detailed knowledge of kinome dynamics, not simply measuring the effect of an inhibitor on one or a few kinases in a pathway.

Most cancers are genetically complex, however, some cancers are physiologically dependent on sustained activity of specific oncogenes, so-called "oncogene addiction" [4]. Oncogene addiction has clear implications for molecular targeting. An example is HER2-driven breast cancer where HER2 is constitutively activated in approximately $20 \%$ of breast cancers. Addiction to HER2 signaling is likely responsible for the strong clinical responses to HER2 inhibitors, and clinical trials and therapeutic response to HER2targeting suggest that HER2 "addiction" defines cell behavior in HER2-driven breast cancer. Thus, HER2-driven (HER2+) breast cancer is an excellent example of a specific cancer subtype in which aberrant signaling of a particular oncogene is key to tumor behavior, and in which strategies targeting HER2 have demonstrated significant efficacy [5]. Lapatinib, a highly selective smallmolecule inhibitor of HER2 and EGFR tyrosine kinase activity, and trastuzumab and pertuzumab, which are humanized monoclonal antibodies to HER2, each have significant clinical benefit in the treatment of HER2+ breast cancer. Despite these highly selective therapeutic molecules that target and inhibit HER2, both clinical and preclinical data suggest that resistance to HER2-targeting results as a consequence of alternate kinase activation (e.g., EGFR, PI3K, IGF1R) [5].

A second example is triple negative breast cancer (TNBC), which includes claudin-low and basal-like breast cancer subtypes [6]. TNBC lacks the current breast cancer targets, including estrogen and progesterone receptors and the receptor tyrosine kinase HER2, therefore chemotherapy remains the mainstay of treatment. Among the most likely candidates for targeted treatment of the basal-like breast cancer TNBC subtype, based on preclinical and correlative 
studies, was the EGFR. For this reason a series of EGFR inhibitor trials were performed, with no more than modest effects in clinical outcome [7, 8]. In one trial of EGFR inhibition in TNBC, gene expression analysis was performed on 16 TNBCs before and one week after beginning therapy with cetuximab, a monoclonal antibody targeting the EGF receptor. Although the EGF receptor pathway was activated in most basallike breast cancers, cetuximab inactivated the downstream MAPK pathway in only a minority, suggesting a more complex kinase signaling network than could be blocked by the single targeted EGF receptor inhibitor [7-9]. Such studies indicate that we need better methods to identify relevant molecular targets or combinations of targets for treatment of TNBC as well as many other cancer types.

These two snapshots of kinome resiliency bypassing targeted inhibition of specific kinases, even in the case of oncogenic addiction with HER2+ breast cancer, underscore our lack of understanding of kinome system signaling. This is true not just in pathological
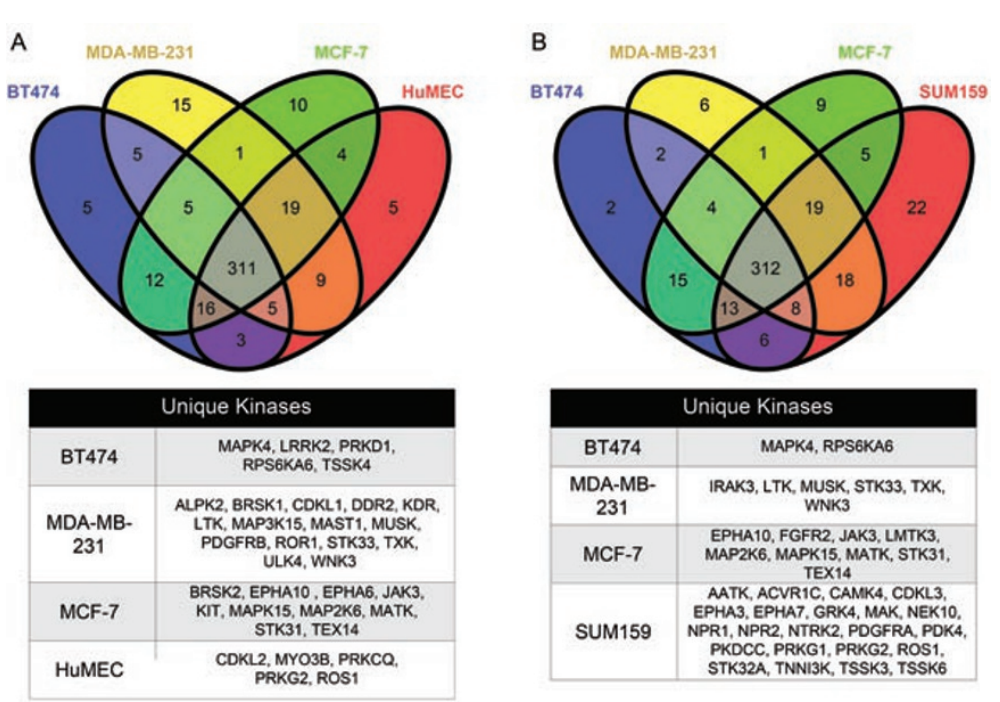

Figure 1 RNA-seq reads for five cell lines were aligned to human genome 19 (HG19) transcripts using Bowtie. The overlap of protein kinases with greater than 50 aligned reads each showed that most kinases were found to be in all cell lines and few kinases were found to be unique to a specific cell line.

conditions such as cancer but in normal cells and tissues as well. Herein we highlight some studies using next-generation deep sequencing to analyze the kinome in breast cancer cell lines.

A largely unanswered question to date is: what is the total kinome expression profile in breast cancer subtypes, including luminal, HER2+ and triple negative? Of the approximate $518 \mathrm{ki}-$ nases in the human kinome, how many kinases are actually expressed in different breast cancer cell lines that are used by investigators around the world?

We used next-generation sequencing to define the expressed kinome in five cell lines: HuMECs (telomerase-immortalized mammary epithelial cells), MCF-7 (luminal), BT474 (HER2+) and two TNBC lines MDA-MB-231 and SUM159 that profile as claudinlow [10]. Figure 1 shows two Venn diagrams comparing the expressed kinases in MCF7, BT474 and MDAMB-231 cell lines relative to HuMECs or SUM159 cells [11]. Each cell type similarly expresses in the range of 375 or so kinases based on a stringent criterion of a minimum of 50 reads in the primary

www.cell-research.com | Cell Research sequence data. This represents nearly $75 \%$ of the kinome expressed at the RNA level. Of the expressed kinases more than $80 \%$ are common in the five cell lines. There are relatively few unique kinases expressed in each cell line. Analysis of the RPKM normalized read counts for BT474 cells shows the expected high expression of ERBB2 (1 744 RPKM normalized read counts in BT474 cells versus 38 in HuMECs). Receptor tyrosine kinases are also the highest expressed kinases in MDA-MB-231 and SUM159 cells, although at significantly lower read counts than ERBB2 in BT474 cells. AXL is expressed at 527 RPKM in MDA-MB-231 cells versus 22 in HuMECs. MET is expressed at 183 RPKM in SUM159 cells versus 107 in HuMECs. Ribosomal S6 kinase (RPS6KB1), a kinase in the mTOR pathway, is interestingly the highest expressed kinase at the transcript level in MCF-7 cells (402 RPKM versus 17 in HuMECs).

If one looks at the 50 most highly expressed kinases in each cell line, they represent each major kinase subfamily within the kinome. There is no significant bias for overrepresentation of any subfamily, including TK, TKL, STE, AGC, CAMK, CMGC and several atypical kinases. This is consistent with the kinome as a functional network in which receptor and cytoplasmic tyrosine kinases are integrated in a coordinate fashion with serine/threonine protein kinases from each subfamily; together they control diverse cellular processes. Further insight is gained from closer examination of the tyrosine kinases expressed in the five cell lines. BT474 cells have high expression of ERBB3 that is the major heterodimerization partner for ERBB2/HER2; this is consistent with ERBB2/ERBB3 signaling as a dominant receptor tyrosine kinase receptor complex in these cells [5]. The understudied Discoidin Domain Receptor 1 (DDR1) is expressed in HuMECs, BT474 and MCF-7 cells, but DDR1 is expressed at extremely low levels in the 
claudin-low cells, MDA-MB-231 and SUM159. In contrast, the two claudinlow TNBC lines significantly express the receptor tyrosine kinases AXL, MET and EGFR, each of which is either absent or expressed at negligible levels in BT474 and MCF-7 cells. It is important to note that HuMECs also express AXL, MET and EGFR. MCF-7 cells highly express significant levels of the IGF1R, which has been shown to be involved in lapatinib resistance $[5,12,13]$. Expression of multiple receptor tyrosine kinases is consistent with MDA-MB-231, SUM159, MCF-7 and HuMECs being highly resistant to growth inhibition by lapatinib. In contrast, lapatinib strongly inhibits growth of BT474 cells because of their addiction to ERBB2. Foretnib, which inhibits AXL and MET, also has little effect on growth of MDA-MB231 and SUM159 cells (unpublished observation), consistent with the ability of alternate receptor tyrosine kinases and downstream signaling pathways controlled by activated oncogenes to promote growth in these cells. This may explain why anti-EGFR therapy or targeting other receptor tyrosine kinases is not sufficient to treat breast cancer patients. It also points out the need to define signaling pathways downstream or parallel to such tyrosine kinases that can be effectively targeted.

Oncogenic mutations downstream of receptor tyrosine kinases in MCF-7 (PI3K), MDA-MB-231 cells (KRAS and BRAF) and SUM159 (HRAS and PI3K) certainly contribute to the resistance to lapatinib or foretinib. However, HuMECs have no defined oncogenic mutations and their growth are not inhibited by lapatinib or foretinib. We also analyzed the mutation status of the SUM159 kinome and found no activating mutations in expressed kinases (unpublished observations). The lack of defined activating kinase mutations or overexpressed kinases in SUM159 cells is consistent with the analysis of a basallike breast cancer primary tumor and metastasis, which had few kinase muta- tions and no obvious kinase gene amplifications [14]. The patient tumor also had no Ras or PI3K mutations. Significant interest is now focused on targeting kinases downstream of receptor tyrosine kinases. The ERK1 and 2 MAPK and PI3K pathways are activated in many cancers and targets of intense drug discovery initiatives $[15,16]$. Focus on ERK1 and 2 is based on the frequent activation of the MEK-ERK pathway in cancer including those with activating Ras mutations. Similarly, PI3K is one of the most commonly mutated genes in cancer and often activates the AKT and mTOR pathways [17]. The primary target for the ERK1/2 pathway has been to develop inhibitors for MEK1 and 2, the upstream kinases that phosphorylate and activate ERK1 and 2. Allosteric inhibitors of MEK1 and 2 have proven to be highly selective because of the unique allosteric regulatory site adjacent to the ATP binding site [2]. Several PI3K inhibitors are currently in clinical trials, as are AKT and mTOR inhibitors that target the principal kinases downstream of PI3K $[16,17]$. The combination of MEK and PI3K inhibitors appears to be of particular efficacy in preclinical models of cancer because two major growth and survival pathways activated in many cancers are inhibited. If tolerated in patients, the dual inhibition of the MEK-ERK and PI3K pathways may prove to be highly effective because these two key pathways promoting growth and survival are both activated by multiple tyrosine kinases. Dual MEK/PI3K inhibition would inhibit oncogenic Ras and PI3K signaling as well. Resistance mechanisms that will arise from dual inhibition of MEK and PI3K are presently undefined.

Determining cancer genomic mutations by the Cancer Genome Atlas Project will define the frequency of mutations and identify new mutations in the kinome and driver oncogenes upstream of specific kinase networks. This database will revolutionize our understanding of the genetic changes in cancer and is identifying new activating and inhibitory mutations in the kinome relevant to specific cancers. One outcome will be the characterization of novel kinase mutations that may provide a tumorigenic advantage but are not common driver mutations. An example is the Tpl2 truncation found in a patient basal-like tumor and metastasis that is predicted to drive MEK-ERK activation [14]. The limitation is that genomic analysis does not define activity of the kinome or kinome response to targeted inhibition. Thus, breakthrough new strategies to define the dynamic activation state of the kinome are needed for future advances.

Recent work by Daub and Mann and co-workers and investigators at Cellzome suggest new quantitative proteomic methods to measure kinome dynamics $[18,19]$. They immobilized kinase inhibitors to Sepharose such that the inhibitor was able to retain binding to kinases in cell lysates. The use of various type I kinase inhibitors allowed the capture of activated versus inactive kinases from lysates of virtually any cell or tissue. By varying the immobilized inhibitor a different repertoire of kinases could be captured. Type I inhibitors represent the majority of inhibitors that have been characterized to date and preferentially recognize the active conformation of kinases. Type I inhibitors are ATP competitive and were generally identified in screens using the activated kinase [2]. In contrast, type II inhibitors recognize the inactive conformation of the kinase and represent many fewer defined inhibitors. Kinases captured by type I inhibitors can be eluted and identified using mass spectrometry. Different quantitative methods such as SILAC, Super SILAC and iTRAQ can be used to quantify the difference in kinase binding to the immobilized inhibitor. For example, the change in kinase binding to the pan kinase inhibitor PP58 covalently coupled to Sepharose can be assayed for kinases from cells treated with or without a growth factor such as 
EGF or an inhibitor such as lapatinib $[18,19]$. Our laboratories have extended this method to use a series of inhibitors, some specific and some very broad pan kinase type I inhibitors, to capture a significant portion of the kinome. Using these methods we have been able to assay the activation state of more than $60 \%$ of the expressed kinome in a single mass spectrometry run. These methods allow the ability to quantitatively measure an unprecedented percentage of the kinome and determine kinome activation state in response to different challenges. The analysis is biased only by the repertoire of immobilized inhibitors used for kinase isolation and can be used with cell lines, preclinical genetically-engineered mouse models of cancer and appropriately handled human tumor specimens.

It is now possible to define the dynamic behavior of the cancer kinome using such quantitative proteomic methods combined with genomic analysis of mutations and expression. A major goal in the coming years will be to understand the resiliency of the kinome to perturbation by targeted kinase inhibition. The resiliency of the kinome is partly a result of "plasticity" of signaling networks comprised of many kinases. Plasticity is the result of kinase networks varying in composition and activity both temporally and spatially in response to different environmental conditions including targeted kinase inhibition. Because of network plasticity, targeting a single kinase may not significantly inhibit network activity (e.g., EGFR in breast cancer). Targeting two kinases such as MEK and PI3K has the ability to significantly inhibit network behavior and arrest signaling in response to different cues (e.g., tyrosine kinases, oncogenic Ras or PI3K). The future promise is to define kinome plasticity in tumor cells responding to single agent inhibitors. Understanding kinome plasticity is necessary for rational design of effective combination therapies to treat cancer in individual patients.

\section{Acknowledgments}

This work was supported by NIH grant GM303024, UNC Breast Cancer SPORE (CA058223) and the University Cancer Research Fund.

\section{References}

1 Walker I, Newell H. Do molecularly targeted agents in oncology have reduced attrition rates? Nat Rev Drug Discov 2009; 8:15-16.

2 Zhang J, Yang PL, Gray NS. Targeting cancer with small molecule kinase inhibitors. Nat Rev Cancer 2009; 9:2839.

3 Fedorov O, Muller S, Knapp S. The (un)targeted cancer kinome. Nat Chem Biol 2010; 6:166-169.

4 Weinstein IB, Joe AK. Mechanisms of disease: Oncogene addiction--a rationale for molecular targeting in cancer therapy. Nat Clin Pract Oncol 2006; 3:448-457.

5 Arteaga CL, Sliwkowski MX, Osborne CK, Perez EA, Puglisi F, Gianni L. Treatment of HER2-positive breast cancer: current status and future perspectives. Nat Rev Clin Oncol 2011; 9:16-32.

6 Prat A, Parker JS, Karginova O, et al. Phenotypic and molecular characterization of the claudin-low intrinsic subtype of breast cancer. Breast Cancer Res 2010; 12:R68.

7 Anders CK, Carey LA. Biology, metastatic patterns, and treatment of patients with triple-negative breast cancer. Clin Breast Cancer 2009; 9 Suppl 2:S73S81.

8 Hoadley KA, Weigman VJ, Fan C, et al. EGFR associated expression profiles vary with breast tumor subtype. BMC Genomics 2007; 8:258.
9 Reeder-Hayes KE, Carey LA, Sikov WM. Clinical trials in triple negative breast cancer. Breast Dis 2010; 32:123136.

10 Langmead B, Trapnell C, Pop M, Salzberg SL. Ultrafast and memory-efficient alignment of short DNA sequences to the human genome. Genome Biol 2009; 10:R25.

11 Oliveros JC. VENNY. An interactive tool for comparing lists with Venn Diagrams. 2007; http://bioinfogp.cnb.csic. es/tools/venny/index.html.

$12 \mathrm{Lu} \mathrm{Y,} \mathrm{Zi} \mathrm{X,} \mathrm{Zhao} \mathrm{Y,} \mathrm{Mascarenhas} \mathrm{D,}$ Pollak M. Insulin-like growth factorI receptor signaling and resistance to trastuzumab (Herceptin). J Natl Cancer Inst 2001; 93:1852-1857.

13 Morgillo F, Woo JK, Kim ES, Hong WK, Lee HY. Heterodimerization of insulin-like growth factor receptor/ epidermal growth factor receptor and induction of survivin expression counteract the antitumor action of erlotinib. Cancer Res 2006; 66:10100-10111.

14 Ding L, Ellis MJ, Li S, et al. Genome remodelling in a basal-like breast cancer metastasis and xenograft. Nature 2010; 464:999-1005.

15 Chappell WH, Steelman LS, Long JM, et al. Ras/Raf/MEK/ERK and PI3K/ PTEN/Akt/mTOR inhibitors: rationale and importance to inhibiting these pathways in human health. Oncotarget 2011; 2:135-164.

16 Ogita S, Lorusso P. Targeting phosphatidylinositol 3 kinase (PI3K)-Akt beyond rapalogs. Target Oncol 2011; 6:103-117.

17 Samuels Y, Wang Z, Bardelli A, et al. High frequency of mutations of the PIK3CA gene in human cancers. Science 2004; 304:554.

18 Daub H, Olsen JV, Bairlein M, et al. Kinase-selective enrichment enables quantitative phosphoproteomics of the kinome across the cell cycle. Mol Cell 2008; 31:438-448.

19 Bantscheff M, Eberhard D, Abraham Y, et al. Quantitative chemical proteomics reveals mechanisms of action of clinical ABL kinase inhibitors. Nat Biotechnol 2007; 25:1035-1044. 